\title{
Diffuse idiopathic skeletal hyperostosis (DISH) is a risk factor for further surgery in short-segment lumbar interbody fusion.
}

\section{$\operatorname{AUTHOR}(\mathrm{S})$ :}

Otsuki, Bungo; Fujibayashi, Shunsuke; Takemoto, Mitsuru; Kimura, Hiroaki; Shimizu, Takayoshi; Matsuda, Shuichi

\section{CITATION:}

Otsuki, Bungo ...[et al]. Diffuse idiopathic skeletal hyperostosis (DISH) is a risk factor for further surgery in short-segment lumbar interbody fusion.. European spine journal 2015, 24(11): 2514-2519

\section{ISSUE DATE:}

2015-11

URL:

http://hdl.handle.net/2433/203072

\section{RIGHT:}

The final publication is available at Springer via http://dx.doi.org/10.1007/s00586-014-3603-5.; The full-text file will be made open to the public on 1 October 2015 in accordance with publisher's 'Terms and Conditions for Self-Archiving',; This is not the published version. Please cite only the published version.; この論文は出版社版でありません。引用の際 には出版社版をご確認ご利用ください。 
Title: Diffuse idiopathic skeletal hyperostosis (DISH) is a risk factor for further surgery in short-segment lumbar interbody fusion

Bungo Otsuki, MD, PhD. ${ }^{1}$

Shunsuke Fujibayashi, MD, PhD. ${ }^{1}$

Mitsuru Takemoto, MD, PhD. ${ }^{1}$

Hiroaki Kimura, MD, PhD. ${ }^{1}$

Takayoshi Shimizu, MD ${ }^{1}$

Shuichi Matsuda, MD, PhD. ${ }^{1}$

${ }^{1}$ Department of Orthopaedic Surgery, Graduate school of medicine, Kyoto university

Please address all correspondence to

Bungo Otsuki

54 Kawahara-cho, Shogoin, Sakyo-ku, Kyoto city, Kyoto, Japan

Phone: (0081)-75-751-3111 
FAX: (0081)-75-751-8409

Email: bungo@kuhp.kyoto-u.ac.jp

Conflicts of Interest and Source of Funding: No conflicts of interest are declared, and no funds were received in support of this work.

This study was performed with the approval of the institutional ethics committee of Kyoto University. 


\section{Abstract}

Purpose To elucidate the effect of diffuse idiopathic skeletal hyperostosis (DISH) on the clinical results of short-segment lumbar interbody fusion (LIF) for the treatment of degenerative lumbar spinal diseases.

Methods The 208 patients who underwent one- or two-level LIF were selected as the subjects of this study. Patients with prior lumbar fusion surgery or follow-up $<1$ year were excluded. Outcome measures were surgery-free survival or the need for further surgery for pseudoarthrosis and/or adjacent segment disease (ASD). The Cox proportional-hazards model were used to identify possible risk factors (DISH, age, sex, number of levels fused, level of lowest instrumented vertebra, and laminectomy adjacent to the index fused levels) for further surgery.

Results Among the 208 patients (39 with DISH), 21 patients required further surgery during follow-up. Cox analysis showed that DISH (hazard ratio = 5.46) and two-level fusion (hazard ratio $=2.83$ ) were significant independent predictors of further surgery. Age, sex, level of lowest instrumented vertebra, 
and laminectomy adjacent to the index fused levels were not significant predictors.

Conclusions DISH after short-segment LIF surgery is a significant risk factor for further surgery because of pseudoarthrosis or ASD.

Key words: diffuse idiopathic skeletal hyperostosis; lumbar interbody fusion; adjacent segment disease; pseudoarthrosis; Cox proportional hazards 


\section{Introduction}

Lumbar interbody fusion (LIF) in conjunction with pedicle screw fixation has become a standard procedure for treating degenerative lumbar spinal diseases with satisfactory clinical results[1, 2]. However, numerous complications and problems have been reported. One major problem is pseudoarthrosis with persistent pain that requires further surgical intervention in some cases. Moreover, with successful fusion, there is also a risk of adjacent segment disease (ASD) and failure as a result of altered biomechanics, and several possible risk factors have been reported in association with ASD[1-3].

Diffuse idiopathic skeletal hyperostosis (DISH) is a skeletal disorder of unknown etiology described in the elderly especially older than 50 years and is characterized by abundant bone formation, ossification, and calcification of connective tissue, especially in the spinal region[4,5]. Bridging ossification occurs most often in the thoracic spine, and nonbridging ossification is characteristic of cervical and lumbar 
involvement[4]. Although many reports have described the effect of DISH on dysphagia or spine trauma[6,7], little is known about the effect of DISH on the treatment of degenerative lumbar spinal diseases.

The thoracolumbar range of motion is restricted in patients with DISH[8]. Therefore, we presumed that the stress concentrated at the fused level or adjacent segment would be greater after fusion surgery in patients with DISH than in patients without DISH because of the longer lever arm, which is associated with a stiff spine. We hypothesized that the failure rate caused by pseudoarthrosis and/or ASD would be higher in patients with DISH than in patients without DISH even after short-segment LIF surgery. The aim of the present study was to determine the effect of DISH on the clinical results of short-segment LIF surgery.

\section{Materials and Methods}

Clinical study design 
This study was performed with the approval of the institutional ethics committee of Kyoto University. The study was a retrospective chart review of 314 consecutive patients who had received one- or two-level LIF surgery for lumbar degenerative diseases performed at our institute from April 2003 through April 2012.

We excluded patients with previous lumbar fusion surgery, Parkinson disease, collagen disease, hemodialysis, active malignancy, lumbar scoliosis (Cobb angle $>30$ degrees), or age $<50$ years, or those with insufficient computed tomography (CT) data for the diagnosis of DISH. Two hundred thirty-one patients met the above criteria. Of these 231 patients, 208 patients were followed for $>1$ year with an average follow-up period of 49.6 months (range: 12-130 months). We analyzed their clinical charts. The clinical outcome was assessed according to the need for further surgery because of pseudoarthrosis and/or ASD in the follow-up period. The influence of possible risk factors on the need for further surgery was examined; these risk factors included sex, age ( $\geq 70$ or $<70$ years), level of 
the lowest instrumented vertebra (S1 or other levels), the number of the fused segments (one or two), laminectomy adjacent to the index fused levels (yes or no), and with or without DISH.

All surgical methods were performed under general anesthesia with a standard open midline approach or the Wiltse approach[9] using a pedicle screw system with one or two interbody cages using standard posterior LIF (PLIF) or transforaminal LIF (TLIF) methods.

Recombinant human bone morphometric protein 2 and other bone-stimulating agents were not used in this study. No patients developed infection in this series.

Definition of DISH

All patients underwent a CT scan from the lower thoracic area to the sacrum before surgery. Ossification of each disk space level from T10 to S1 was assessed by the lateral lumbar X-ray, reconstructed sagittal CT view, 
and then graded according to the Mata scoring system[10]. Each vertebral level was scored as follows: 0) no ossification, 1) ossification without bridging, 2) ossification with incomplete bridging, and 3) ossification with complete bridging of the disk space. We defined DISH as the presence of ossification of grade 2 or 3 along the anterolateral aspect of three contiguous disk spaces with relative preservation of disk height according to the Resnick criteria[5].

Using these criteria, we classified the patients into two groups: DISH+ and DISH-. DISH was diagnosed by the senior author (BO). To evaluate interobserver reliability of the radiological assessments of DISH, another independent orthopedic surgeon (TS) also reviewed the last 70 consecutive cases blindly and independently, and these results were compared with those of the senior author.

Statistical analyses 
Age and follow-up duration were compared between DISH+ and

DISH- groups using a two-tailed $t$ test and Mann-Whitney $U$ test, respectively. Fisher's exact test was used to compare the rate of index pathology, the number of levels fused, and the rate of further surgery between the DISH+ and DISH- groups. Survival curves were produced using the Kaplan-Meier method. To evaluate the influence of possible risk factors on the need for further surgery, the log-rank test and proportional-hazards modeling were used in univariate and multivariate analysis, respectively. All factors in the univariate analysis were included in the multivariate analysis using backward stepwise selection of covariates. The proportional hazards assumption was checked for each explanatory factor by the method described previously[11], and a $P$ value $<0.05$ was considered significant evidence that the assumption was violated by a given factor. All explanatory factors in our Cox model passed the test, suggesting that the proportional hazards assumption was reasonable. A $P$ value $<0.05$ 
was considered significant in all analyses. All calculations were performed using $\mathrm{R}$ ( $\mathrm{R}$ for 3.1.0 GUI 1.64).

\section{Results}

Patients’ characteristics

A total of 208 patients (99 men and 109 women) underwent one- or two-level LIF surgery; their mean age was 67.7 years (range: 50-86). Among the 208 patients, 181 patients were treated with TLIF and 27 patients were treated with PLIF. In the follow-up period, 21 patients were treated with further surgery because of pseudoarthrosis and/or ASD. The results of the Kaplan-Meier analysis for all patients are shown in Fig. 1. The KaplanMeier survivorship analysis predicted 5- and 10-year prevalence rates of $12.1 \%(95 \%$ confidence interval $[\mathrm{CI}]=7.4-19.5)$, and $24.6 \%(95 \% \mathrm{CI}=$ 12.9-44.6), respectively. 
Treatment and clinical results in the DISH+ and DISH- groups

Among the 208 patients, 39 patients were diagnosed with DISH (DISH+). In the test of interobserver reliability, the kappa coefficient was 0.89, which indicated significant high reliability for the two independent observers. Details of the baseline data are shown in Table 1 . The mean age was significantly higher in the DISH+ than in the DISH- group, and the prevalence of DISH was significantly higher in men than in women. However, the index pathology, number of levels fused, rate of laminectomy adjacent to the index fused levels, and level of lowest instrumented vertebra did not differ significantly between the DISH+ and DISH- groups.

The rate of further surgery in the follow-up period was significantly higher in the DISH+ than in the DISH- group.

The effect of DISH and other possible risk factors on the need for further surgery 
The univariate analysis using the log-rank test showed that DISH was a significant risk factor for further surgery $(P<0.05)$. Male sex, two-level fusion, and receiving a laminectomy adjacent to the index fused levels were borderline significant risk factors $(P<0.15)$ for further surgery (Table 2).

Multivariate analysis using the Cox proportional-hazards model revealed that DISH $(P=0.00017)$ and the number of levels fused $(P=0.029)$ were significant independent predictors of the need for further surgery, whereas other factors were rejected as independent contributors (Table 2). The hazard ratio for needing further surgery was 5.46 times higher in the DISH+ group compared with the DISH- group. The results of the Cox modeling of DISH and the number of levels fused on survival are shown in Fig. 2.

\section{Discussion}


DISH has been investigated in relation to dysphagia[6,12], dyspnea[13], spine trauma[7,14], and metabolic diseases[15], but little is known about the clinical effects of DISH on degenerative lumbar spine diseases. Although the intervertebral disk space is usually preserved in patients with DISH, the condition may coexist with degenerative disk disease[16]. Chi et al. [17] reported on the successful case report of L2/3 posterior fusion for degenerative change of L2/3, which was caudally adjacent level of contiguous ossification from C2 to L2. To our knowledge, no other report has described the treatment of lumbar degenerative disease complicated by DISH.

The current study is the first to focus on the relationship between DISH and the clinical outcomes of LIF surgery. In the analyses of 208 patients who received a short-segment LIF, the rate of further surgery was significantly higher in DISH+ group (25.6\%) than in DISH- group (6.5\%) during follow-up periods. Moreover, the univariate log-rank test also showed that DISH was a significant risk factor for the need for further 
surgery $(P=0.00013)$. However, the baseline age and sex distribution differed between the DISH+ and DISH- groups (Table 1). Several factors, including age, sex, level of lowest instrumented vertebra, laminectomy adjacent to the index fused levels, and the number of levels fused, have been reported as risk factors for ASD[2,3,18]. Therefore, we selected these factors as potential confounding risk factors and included these factors together with DISH into the Cox proportional-hazards model. DISH and the number of levels fused were identified as independent risk factors, with hazard ratios for further surgical intervention of 5.46 and 2.83, respectively.

The reason for the worse clinical results in $\mathrm{DISH}^{+}$patients is presumed as follows. The ossification in DISH is usually first seen in the thoracic region and extends to the lumbar region with age[10]. The average flexion-bending ranges of motion between T11 and L2 in healthy subjects has been reported as 18.7 degrees[19]. Patients with DISH lose the flexibility of the thoracolumbar junction[8], which may result in concentration of stress in the lumbar region. After a fusion surgery, the 
length of the rigid sections increases further, so that the stress to the pedicle screw may be greater in patients with than in those without DISH. In some cases, severe instability of the index fusion levels leads to pseudoarthrosis at the index fusion levels. Even if a solid fusion can be acquired in the index fusion levels, the stress to the adjacent levels will be greater in patients with than in those without DISH, which may lead to severe ASD in patients with DISH.

As described previously, several risk factors have been reported to affect the clinical results of lumbar spine fusion surgery. Besides DISH, the number of levels fused was shown to be a risk factor for further surgery in this study. In a retrospective review of 106 lumbar fusions, Gillet et al. [20] noted that the rate of further surgery increased as the number of levels fused increased. The same trend was also confirmed by Sears[2] for 912 lumbar fusions. However, Ghiselli et al. [18] did not find a significant association between the number of levels fused and the subsequent development of ASD, and the issue remains controversial. 
Increasing age is a risk factor for ASD[2,21]. However, age was not identified as an independent risk factor for further surgery in this study. The rates of comorbidity and surgical complications of spine surgery have been reported to increase with age[22]. We might hesitate to perform further surgery on older patients with several comorbidities even in those with clinically apparent ASD or pseudoarthrosis.

The issue of "floating" fusion remains controversial in the literature[2,23]. Although the present study did not find a significantly higher relative risk for fusion ending at L3, L4, or L5 compared with S1, the result might be different for longer-segment fusion surgery.

Apart from ASD and pseudoarthorosis, we also recorded the incidence rate of the vertebral fractures of the lower thoracic and lumbar spine after the surgery. In the follow-up period, ten out of 169 DISH-group and two out of $39 \mathrm{DISH}+$ group suffered from osteoporotic vertebral fracture. The incidence rate was not statistically different between two groups. One patient in DISH- group and one patient in DISH+ group 
underwent vertebroplasty using polymethylmethacrylate cement.

There are several limitations in the present study. The first is the definition of DISH. In the current study, the existence of DISH was diagnosed using lateral lumbar X-ray and a reconstructed sagittal CT view in which the lower thoracic spine to the sacrum was scanned. Therefore, we defined the criteria for DISH as an ossification of $\geq 4$ contiguous vertebral levels from T10 to S1; that is, "thoracolumbar" or "lumbar" DISH[24]. Therefore, three lower contiguous levels of ossification entirely in the thoracic region, namely "thoracic" DISH[24], could not be diagnosed. Further study is needed to examine the effect of "thoracic" DISH on the outcomes of LIF surgery. The second limitation is that we chose the endpoint of further surgery for pseudoarthrosis and/or ASD rather than attempting to retrospectively identify and quantify clinically significant (symptomatic) ASD and/or pseudoarthrosis. Simplifying the methodology this way carries the risk of underestimating the rate of clinically significant pseudoarthrosis and/or ASD because some patients with symptomatic pseudoarthrosis and/or 
ASD may be excluded for various reasons, such as intercurrent illness or patient preference. The small number of patients needing further surgery and the possible risk factors selected are other limitations. Only 21 of 208 patients were given further surgery, and we could select only five possible risk factors besides DISH. The patients' characteristics were heterogeneous in terms of implants type, size or material properties of the LIF cage, spinal balance before and after surgery, and bone mineral density. Further studies should include larger cohorts using various fusion techniques to gain a better understanding of various risk factors including DISH. Finally, the average follow-up period was about 50 months and was not enough for the precise evaluation of the clinical results after LIF surgery. We should evaluate the clinical data again several years later.

Although the detailed mechanism is unclear, DISH has been shown to be a significant risk factor for the need for further surgery after short-segment LIF. Surgeons should notice the existence of DISH when performing LIF surgery, even if ossification is seen only in the thoracic to 
upper lumbar region. 


\section{References}

1. Greiner-Perth R, Boehm H, Allam Y, et al. Reoperation rate after instrumented posterior lumbar interbody fusion: a report on 1680 cases. Spine 2004;29:2516-20.

2. Sears WR, Sergides IG, Kazemi N, et al. Incidence and prevalence of surgery at segments adjacent to a previous posterior lumbar arthrodesis. The spine journal : official journal of the North American Spine Society 2011;11:11-20.

3. Etebar S, Cahill DW. Risk factors for adjacent-segment failure following lumbar fixation with rigid instrumentation for degenerative instability. Journal of neurosurgery 1999;90:163-9.

4. Forestier J, Lagier R. Ankylosing hyperostosis of the spine. Clinical orthopaedics and related research 1971;74:65-83.

5. Resnick D, Niwayama G. Radiographic and pathologic features of spinal involvement in diffuse idiopathic skeletal hyperostosis (DISH). Radiology 1976;119:559-68. 
6. Mosher HP. Exostoses of the cervical vertebrae as a cause for difficulty in swallowing. The Laryngoscope 1926;36:181-2.

7. Diederichs G, Engelken F, Marshall LM, et al. Diffuse idiopathic skeletal hyperostosis (DISH): relation to vertebral fractures and bone density. Osteoporosis international : a journal established as result of cooperation between the European Foundation for Osteoporosis and the National Osteoporosis Foundation of the USA 2011;22:1789-97.

8. Al-Herz A, Snip JP, Clark B, et al. Exercise therapy for patients with diffuse idiopathic skeletal hyperostosis. Clinical rheumatology 2008;27:207-10.

9. Wiltse LL. The paraspinal sacrospinalis-splitting approach to the lumbar spine. Clinical orthopaedics and related research 1973:48-57.

10. Mata S, Chhem RK, Fortin PR, et al. Comprehensive radiographic evaluation of diffuse idiopathic skeletal hyperostosis: development and interrater reliability of a scoring system. Seminars in arthritis and rheumatism 1998;28:88-96. 
11. Grambsch PM, Therneau TM. Proportional hazards tests and diagnostics based on weighted residuals. Biometrika 1994;81:515-26.

12. Carlson ML, Archibald DJ, Graner DE, et al. Surgical management of dysphagia and airway obstruction in patients with prominent ventral cervical osteophytes. Dysphagia 2011;26:34-40.

13. Mader R. Clinical manifestations of diffuse idiopathic skeletal hyperostosis of the cervical spine. Seminars in arthritis and rheumatism $2002 ; 32: 130-5$.

14. Bransford RJ, Koller H, Caron T, et al. Cervical spine trauma in diffuse idiopathic skeletal hyperostosis: injury characteristics and outcome with surgical treatment. Spine 2012;37:1923-32.

15. Mader R, Novofestovski I, Adawi M, et al. Metabolic syndrome and cardiovascular risk in patients with diffuse idiopathic skeletal hyperostosis.

Seminars in arthritis and rheumatism 2009;38:361-5.

16. Mader R, Dubenski N, Lavi I. Morbidity and mortality of hospitalized patients with diffuse idiopathic skeletal hyperostosis. 
Rheumatology international 2005;26:132-6.

17. Chi D, Miyamoto K, Hosoe H, et al. Symptomatic lumbar mobile segment with spinal canal stenosis in a fused spine associated with diffused idiopathic skeletal hyperostosis. The spine journal : official journal of the North American Spine Society 2008;8:1019-23.

18. Ghiselli G, Wang JC, Bhatia NN, et al. Adjacent segment degeneration in the lumbar spine. The Journal of bone and joint surgery. American volume 2004;86-A:1497-503.

19. Gercek E, Hartmann F, Kuhn S, et al. Dynamic angular three-dimensional measurement of multisegmental thoracolumbar motion in vivo. Spine 2008;33:2326-33.

20. Gillet P. The fate of the adjacent motion segments after lumbar fusion. Journal of spinal disorders \& techniques 2003;16:338-45.

21. Min JH, Jang JS, Jung B, et al. The clinical characteristics and risk factors for the adjacent segment degeneration in instrumented lumbar fusion. Journal of spinal disorders \& techniques 2008;21:305-9. 
22. Mannion AF, Fekete TF, Porchet F, et al. The influence of comorbidity on the risks and benefits of spine surgery for degenerative lumbar disorders. European spine journal : official publication of the European Spine Society, the European Spinal Deformity Society, and the European Section of the Cervical Spine Research Society 2014;23 Suppl 1:S66-71.

23. Disch AC, Schmoelz W, Matziolis G, et al. Higher risk of adjacent segment degeneration after floating fusions: long-term outcome after low lumbar spine fusions. Journal of spinal disorders \& techniques $2008 ; 21: 79-85$.

24. Holton KF, Denard PJ, Yoo JU, et al. Diffuse idiopathic skeletal hyperostosis and its relation to back pain among older men: the MrOS Study. Seminars in arthritis and rheumatism 2011;41:131-8. 


\section{Figure legends}

Fig. 1. Kaplan-Meyer analysis of further surgery in all patients. The dotted lines show the $95 \%$ confidence interval.

Fig. 2. Cox proportional-hazards regression survival curve for A, DISH

(stratified into DISH+ and DISH- groups) and B, the number of levels fused (stratified into one-level fusion and two-level fusion). 
Fig. 1

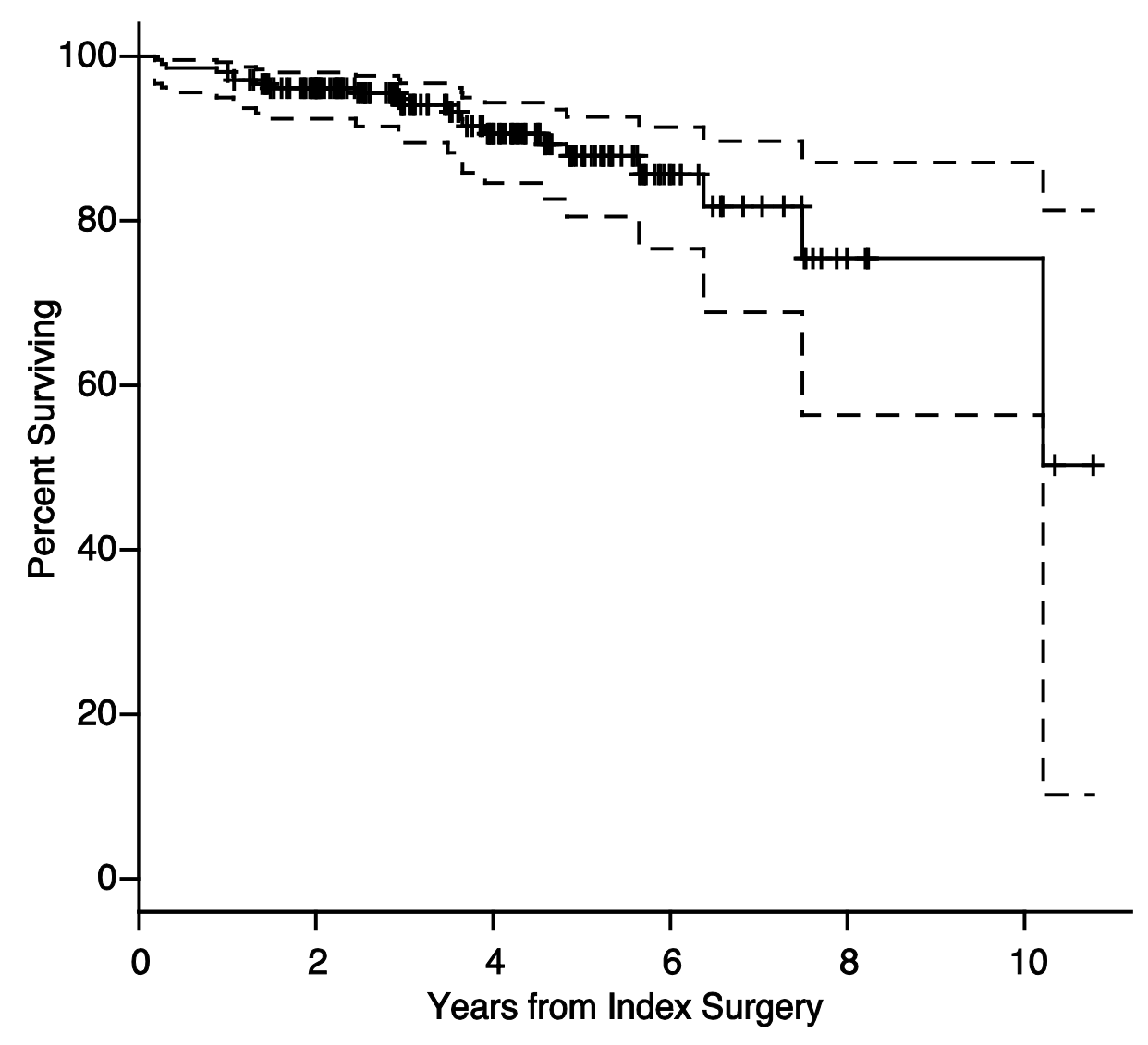


Fig. 2

Survival curve adjusted for DISH

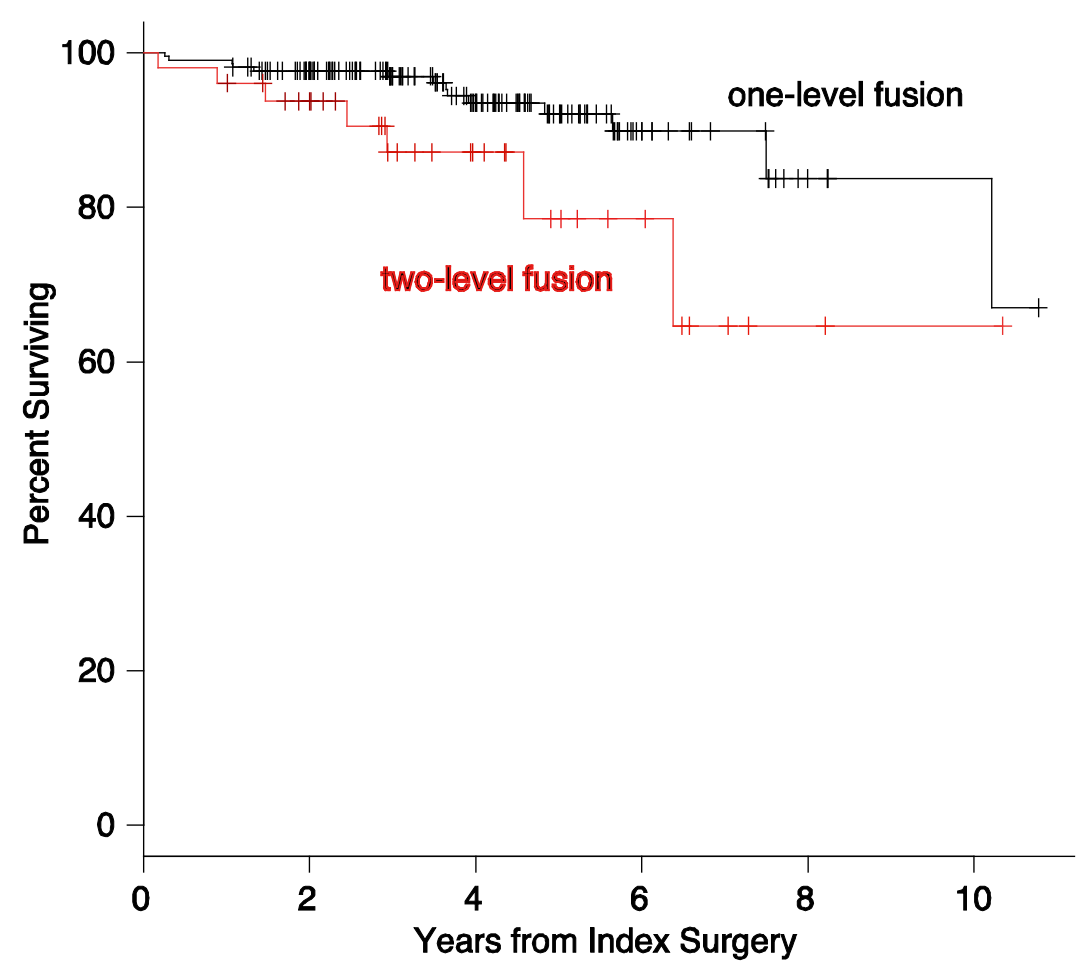

Survival curve adjusted for the number of levels fused

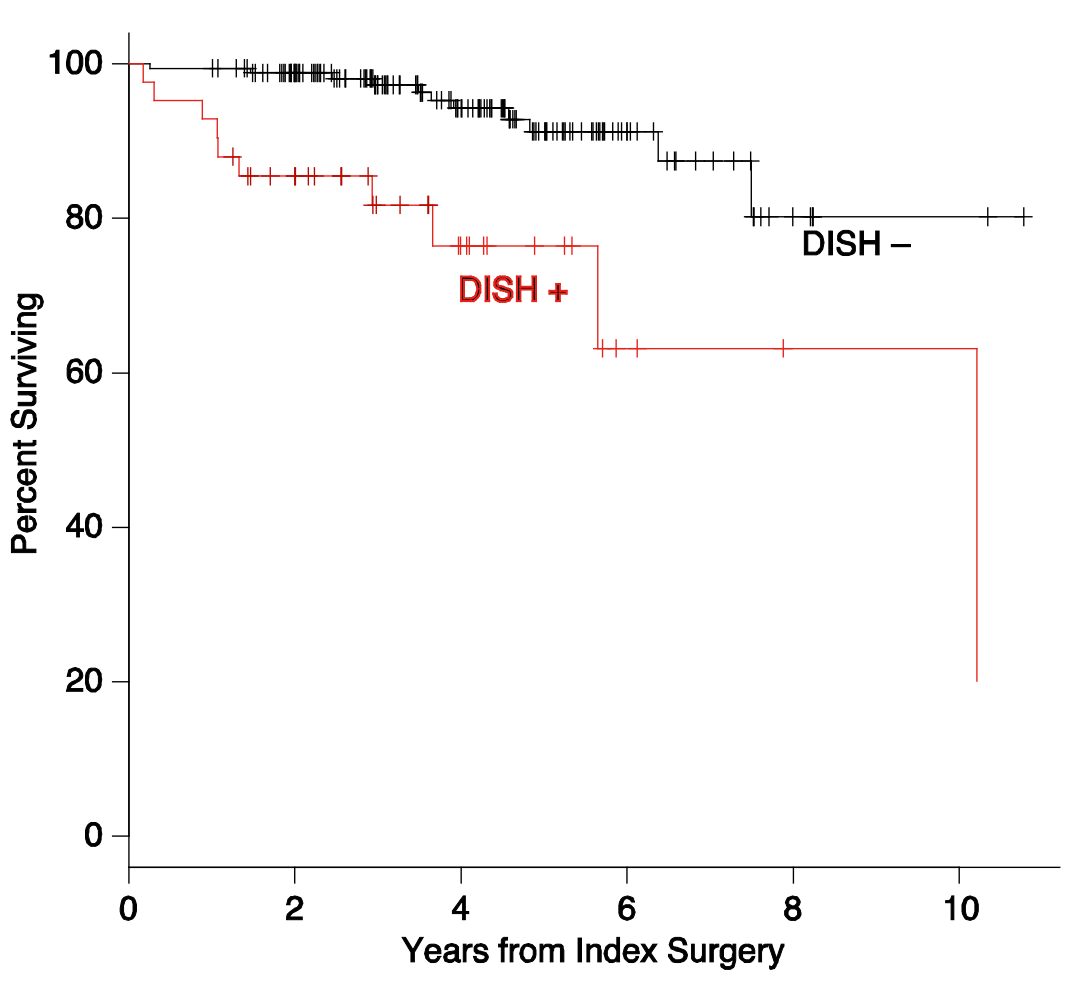


TABLE 1 Demographic data and clinical results in the DISH+ and DISH- Groups

\begin{tabular}{|c|c|c|c|}
\hline & DISH+ & DISH- & $P$ \\
\hline No. of patients & 39 & 169 & \\
\hline Mean age \pm SD (years) & $71.4 \pm 6.82$ & $66.9 \pm 8.36$ & $0.0021^{\mathrm{a}}$ \\
\hline \multicolumn{4}{|l|}{ Sex } \\
\hline Male & 29 & 70 & \multirow{2}{*}{$0.00030^{\circ}$} \\
\hline Female & 10 & 99 & \\
\hline Follow-up period \pm SD (months) & $46.6 \pm 25.5$ & $50.3 \pm 22.8$ & 0.37 \\
\hline Index pathology & \multicolumn{2}{|c|}{ (Not mutually exclusive) } & \\
\hline Lumbar spinal canal stenosis & 20 & 60 & 0.10 \\
\hline Degenerative spondylolisthesis & 14 & 82 & 0.21 \\
\hline Lytic spondylolisthesis & 4 & 19 & 1.00 \\
\hline Degenerative disk disease & 2 & 14 & 0.74 \\
\hline Degenerative scoliosis & 1 & 9 & 0.69 \\
\hline Disk hernia & 6 & 17 & 0.39 \\
\hline One-level fusion & $31(79.5 \%)$ & $139(82.2 \%)$ & \\
\hline $\mathrm{L} 2 / 3$ & 1 & 4 & \\
\hline $\mathrm{L} 3 / 4$ & 4 & 10 & \\
\hline $\mathrm{L} 4 / 5$ & 19 & 92 & \\
\hline $\mathrm{L} 5 / \mathrm{S}$ & 7 & 27 & 0.65 \\
\hline Two-level fusion & $8(20.5 \%)$ & 30 (17.8\%) & \\
\hline $\mathrm{L} 2 / 3 / 4$ & 1 & 0 & \\
\hline $\mathrm{L} 3 / 4 / 5$ & 3 & 26 & \\
\hline $\mathrm{L} 4 / 5 / \mathrm{S}$ & 4 & 10 & \\
\hline $\begin{array}{l}\text { Laminectomy adjacent to the index } \\
\text { fused levels }\end{array}$ & 15 (38.5\%) & 42 (24.9\%) & 0.11 \\
\hline
\end{tabular}

\begin{tabular}{lccc}
\hline $\begin{array}{l}\text { Level of lowest instrumented } \\
\text { vertebra }\end{array}$ & & & \\
S1 & 11 & 37 & 0.40 \\
L3, L4, or L5 & 28 & 132 & $0.0014^{\mathrm{a}}$ \\
\hline Further surgery & $10(25.6 \%)$ & $11(6.5 \%)$ & \\
\hline ASD & 7 & 7 & 1.0 \\
Pseudoarthrosis & 2 & 2 & \\
ASD + pseudoarthrosis & 1 & 2 & \\
\hline
\end{tabular}

DISH, diffuse idiopathic skeletal hyperostosis; $A S D$, adjacent segment disease; $S D$, standard deviation a, $P<0.05$ 
TABLE 2 Relationships between potential risk factors and survival

\begin{tabular}{|c|c|c|c|c|}
\hline \multicolumn{5}{|c|}{ Univariate analysis } \\
\hline \multicolumn{2}{|l|}{ Factors } & Variables (number) & Further surgery (\%) & $P$ \\
\hline \multirow{2}{*}{\multicolumn{2}{|c|}{ Sex }} & Male (99) & $15(15.2)$ & \multirow{2}{*}{$0.10^{b}$} \\
\hline & & Female (109) & $6(5.5)$ & \\
\hline \multirow{2}{*}{\multicolumn{2}{|c|}{ Age }} & $<70(113)$ & $12(10.6)$ & \multirow{2}{*}{0.90} \\
\hline & & $\geq 70$ (95) & $9(9.5)$ & \\
\hline \multirow{2}{*}{\multicolumn{2}{|c|}{$\begin{array}{c}\text { Level of lowest } \\
\text { instrumented vertebra }\end{array}$}} & S1 (48) & $6(12.5)$ & \multirow{2}{*}{0.20} \\
\hline & & L3, L4, or L5 (160) & $15(9.3)$ & \\
\hline \multirow{2}{*}{\multicolumn{2}{|c|}{$\begin{array}{c}\text { Number of levels } \\
\text { fused } \\
\end{array}$}} & One (170) & $14(8.2)$ & \multirow{2}{*}{$0.09^{b}$} \\
\hline & & Two (38) & $7(18.4)$ & \\
\hline \multirow{2}{*}{\multicolumn{2}{|c|}{$\begin{array}{l}\text { Laminectomy } \\
\text { adjacent to the index } \\
\text { fused levels }\end{array}$}} & Yes (57) & $7(12.0)$ & \multirow[b]{2}{*}{$0.13^{b}$} \\
\hline & & No (151) & $14(9.3)$ & \\
\hline \multirow{2}{*}{\multicolumn{2}{|c|}{ DISH }} & DISH+ (39) & $10(25.6)$ & \multirow{2}{*}{$0.00013^{\mathrm{a}}$} \\
\hline & & DISH- (169) & $11(6.5)$ & \\
\hline \multicolumn{5}{|c|}{ Multivariate analysis } \\
\hline Factors & & Hazard ratio & $95 \%$ CI & $P$ \\
\hline DISH & & $6(\mathrm{DISH}+$ vs DISH-) & $2.25-13.3$ & $0.00017^{\mathrm{a}}$ \\
\hline $\begin{array}{l}\text { Number of levels } \\
\text { fused }\end{array}$ & 2.8 & (two-level vs one-level) & $1.11-7.20$ & $0.029^{a}$ \\
\hline
\end{tabular}

